\title{
Osteonecrosis of the Femoral Head : Could oxygen- ozone therapy became a treatment option?
}

\author{
Iliakis $\mathrm{E}^{1}$, Bonetti $\mathrm{M}^{2}$, lliakis $\mathrm{A}^{3}$
}

${ }^{1}$ Orthopaedic Surgeon. Ozonaim Clinic, Athens, Greece, ${ }^{2}$ Dept. of Neuroradiology - Istituto Clinico Città di Brescia, Brescia, Italy, ${ }^{3}$ Veterinary Clinic. Citta di Pavia. Pavia. Italy.

doi: 10.7203/jo3t.1.1.2015.12163 Received: October, $2015 \quad$ Published: December 2015

\section{ABSTRACT}

Osteonecrosis of the femoral head $(\mathrm{ONFH})$ is caused by inadequate blood supply leading to death of the osteocytes. Subsequently it progresses to collapse of the femoral head and advanced joint destruction.

Medical Ozone (MO) causes an increase in the red blood cell glycolysis rate. This leads to the stimulation of 2,3-diphosphoglycerate which leads to an increase in the amount of oxygen released to the tissues. Production of prostacyline, a vasodilator, is also induced by $\mathrm{O}_{3}$.

Intra-articular injection of M.O. is often proposed as a conservative treatment for osteoarthritic pain particularly in the knees because it is less expensive or aggressive than other methods, like Hyaluronic acid and total knee replacement.

Therefore, it is important to evaluate for the possibility to apply the method at first intraarticularly in hips and then to document its efficacy to patients with avascular necrosis of the femoral head. We prefer to use ultrasound images that ensure the safety and the precision of the injection.

We treated a 43 years old female patient with a dominant pain in the left hip and to a lesser extent to the sacroiliac joint for approximately 3 months, grade III Steinberg after previous Hyperbaric oxygen therapy (HBO) before. Our treatment was weekly for seven times, intraarticularly, at volume $5 \mathrm{ml}$ and concentration $40 \mathrm{mgr} \mathrm{O} 3 / \mathrm{ml} \mathrm{O} 2$ and in the upper posterior edge $3 \mathrm{ml}$ of $\mathrm{MO}$ in the same concentration. The evaluation with magnetic resonance imaging (MRI) revealed improvements after the MO treatment and the clinical status gradually improved during the treatment with the patient walking without assistance after the final injection, pain free.

The positive outcome of the first case of ONFH treated with intraarticular MO injections led us to believe that this method values further studies.

Keywords: Osteonecrosis femoral head oxygen ozone therapy

\section{INTRODUCCIÓN}

Ozone therapy is not such a well-known medical method in the academic community although it is practiced by a fair number of physicians around the world. It is mainly recommended to patients with low back pain $(1,2)$, inflammatory diseases of the knees $(3,4)$ and to deal with symptoms of the diabetic foot $(5,6,7)$. Various studies have been published explaining the biochemical mechanism of action of the medical ozone therapy against the oxidizing stress (8) and the improvement of the circulation $(9,10)$. Taking under consideration the

Authors Information

Email:driliakis@hotmail.com above said combined with the low cost and lack of side effects (11), this type of treatment was proposed for this young patient. The potential effectiveness of the ozone therapy in the ONFH a condition in which traditional medical approaches are not very effective and the threat of surgery is high $(12,13)$, is the argument of this study.

Osteonecrosis of the femoral head (ONFH) is the pathologic consequence when inadequate blood supply of the area leads to death of the osteocytes and subsequently collapse of the articular surface and joint destruction.

Patients that suffer from ONFH are generally young adults ( 35 to 45 years old) and risk factors for the majority of cases include alcohol, smoking, chronic steroid use, hip trauma including femoral neck fractures and hip dislocations, and prior hip 
surgery. Other potential causes of osteonecrosis include deep sea diving or other hyperbaric conditions, systemic lupus erythematosus (SLE) and other connective tissue disorders, autoimmune diseases causing vasculitis, sickle cell anemia, coagulopathy such as thrombophilia or disseminated intravascular coagulation, human immunodeficiency virus (HIV) infection, hyperlipidemia, fat embolus syndrome, treatment of developmental hip dysplasia, chemotherapy and/or radiation, organ transplantation, chronic liver disease, Gaucher disease, gout, and metabolic bone disease (14). Estimates suggest that up to 20,000 new cases of AVN are diagnosed each year in the United States alone $(15,16)$ and up to $60 \%$ occur bilaterally (17).

\section{Clinical findings}

ONFH may be asymptomatic and is usually discovered incidentally on radiographs .However, it can become painful with limitation of hip range of movement with advancement of disease. Patients with AVN of the femoral head often report groin pain that is exacerbated by weight bearing. The pain may initially be mild but progressively worsens over time and with use. Eventually, the pain is also present at rest and during the night.

\section{Anatomical findings.}

Regardless of the underlying etiology of osteonecrosis, several studies suggest a common pathogenic pathway involving apoptosis of osteoblasts and osteocytes $(18,19,20)$. Due to infarction, oxygen and nutrient deprived osteocytes and marrow cells die. As the collateral circulation supplying the epiphyses is limited, capillary arterialization may not restore sufficient blood flow to the tissues (21). Added to this vascular shortage and programmed cell death, defective bone repair plays an equally important role in the pathogenesis of osteonecrosis (22). Adipogenesis is another causal factor in steroid and alcohol related osteonecrosis, as it leads to the compression of venous sinusoids and congestion. The venous congestion increases intraosseous pressure, preventing adequate arterial blood flow, eventually leading to bone infarction $(23,24)$. Finally, genetic factors, such as mutations in the COL2Al gene, have been associated with the pathogenesis of osteonecrosis (25).

\section{Classification}

The main classification system describing the clinical and radiological severity/progression of ONFH is the Steinberg system from the University of Pensilvania (26). Although various other systems have been proposed.

\section{Radiologic findings}

$\mathrm{X}$-ray findings of ONFH take at least 2 months to develop, but may take as long as 6 months. Sclerosis and cystic changes are early radiographical changes. With progression of the disease the femoral head collapses losing its spherical structure and the joint space is reduced (secondary arthritis).

MRI is the most sensitive diagnostic tool for ONFH as it has sensitivity of $90-100 \%$ and specificity of $100 \%$ in diagnosis. It is also useful for early detection of asymptomatic. The ONFH characteristic appearance of the infarcted area is a hypo-dense on T1 image surrounded by a single hypo-dense line separating normal and osteonecrotic bone. T2 image shows another line within this line representing increased vascularity in granulation tissue. The appearance of the interface is more important in the diagnosis, and the density of the necrotic central part will change with the change in fat content due to death of adipocytes and appearance of reparative tissue. MRI can help in identifying patients at risk of collapse of the femoral head. Presence of bone marrow edema, increased fat content in the proximal femur and joint effusion on MRI are important prognostic factors (27). Dynamic MRI may be the future investigation for early prediction of vascular insult to femoral head.

Studies have shown that bone scintigraphy of the hip has lower resolution and sensitivity in the diagnosis of osteonecrosis compared to MRI (28).

\section{Ozone.}

A complete description of the biochemical mechanism of action of ozone was reported in works of Bocci, Sagai, Borelli, Elvis and al (29, $30,3,31$ ). For our case is usuful this (3): Briefly ozone dissolves physically in pure water and in biological water (physiological saline, plasma, lymph, urine) according Henry's law in relation to the temperature, pressure and ozone concentration. During the major ozonated autohaemotherapy, for example, ozone immediately reacts with plasmatic antioxidants, polyunsaturated fatty acids (PUFA), proteins and carbohydrates. Thus, ozone leads to the formation of Reactive oxygen species (ROS), lipid oxidation products (LOP) and oxidized antioxidant producing immediately two crucial messengers: hydrogen peroxide, one of the reactive oxygen species, and aldehydes such as 4hydroxy-2-nonenal (4-HNE) as the result of PUFA 
peroxidation. $\mathrm{H} 2 \mathrm{O} 2$ is a common compound in blood cells and, by entering into the mass of erythrocytes activates glycolysis with a transient increase of ATP and of 2-3-diphosphoglycerate (2,3-DPG). This compound causes a shift to the right of oxyhemoglobin and this very useful process improves the release of oxygen into ischemic tissues, an important step in ischemic diseases. It also causes a significant reduction in $\mathrm{NADH}$ and helps to oxidize cytochrome $\mathrm{C}$. There is a stimulation of production of enzymes which act as free radical scavengers and cell-wall protectors: glutathione peroxidase, catalase and superoxide dismutase. Production of prostacyline, a vasodilator, is also induced by $\mathrm{O}_{3}$ (32).

\section{CASE PRESENTATION}

A female patient (43 years old) visited our private practice with a three month history of pain of the left hip. The patient referred that the pain came up one day after four hours of continuous bending during work. Non direct traumatic injury was reported. At examination her pain was pretty severe; rated $8 / 10$ on numerical rating scale with groin pain as well as pain in the anterior part of the thigh. Less pain mainly induced by pressure $(5 / 10)$ presented in both posterior sacroiliac joints (more in the left). Furthermore, the compression of the iliac crest, superior posterior iliac spine, iliolumbar ligaments, both piriformis muscles and supraspinal ligaments was painful. Twisting the left thigh in abduction was extremely painful while the right thigh was pain free and moved freely. Contracture of the left lower limb (20 degrees) with intense pain mainly during extension while there was limitation in the abduction (10 degrees) and to a lesser extent limitation in adduction (30 degrees). No examination procedure produced any pain in the right leg. The patient walked with handles.

\section{Health history.}

She is a tall person of low weight, smoking (10 cigarettes/day), hypercholisterolemia (270 ), no alcohol consumption and free of any current medication, only painkillers like paracetamol with codeine. The patient presented to us with one Xray and two MRI tests. The X-ray did not reveal any notable degenerative changes of the lumbar spine or of the sacroiliac joints. The first MRI (fig 1) was performed about two months before and the
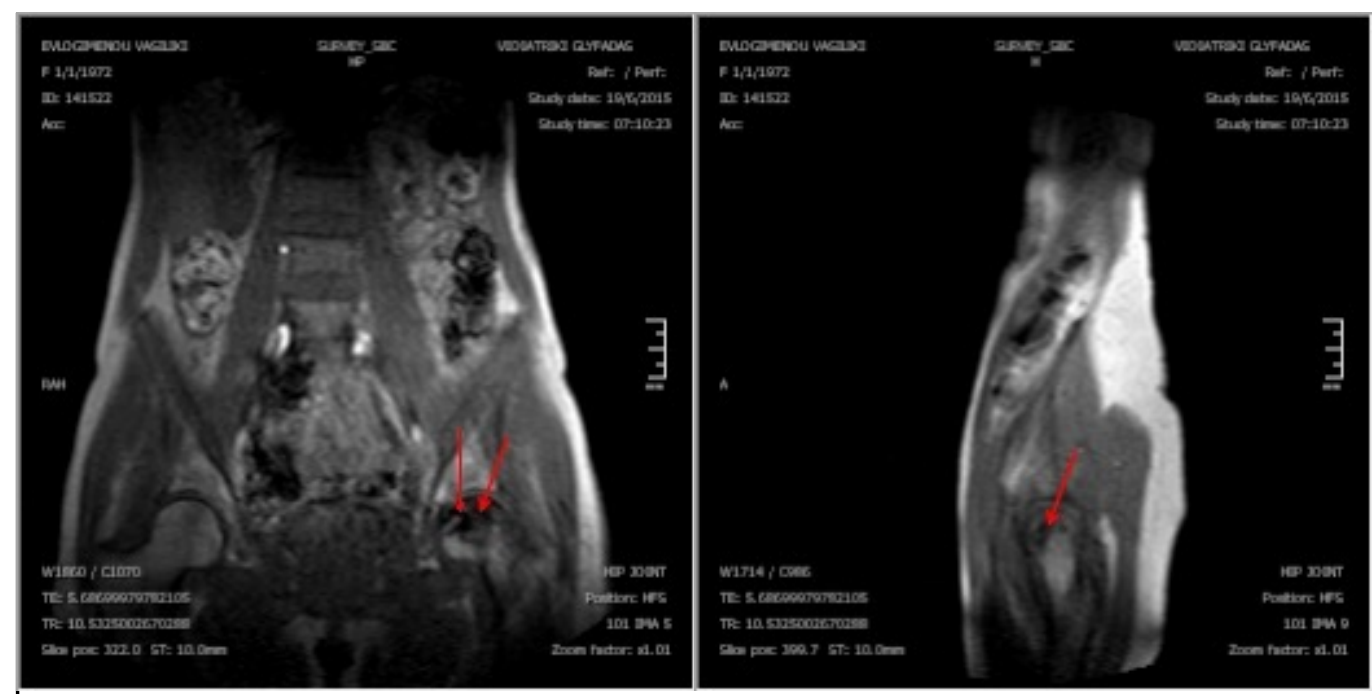

Fig. 1

Sizeable distortion of irregular shape of the subchondral surface of the left femoral head. The characteristic appearance of the infarcted area is a hypo-dense image surrounded by a single hypo-dense line separating normal and osteonecrotic bone (arrows). T2 image shows another line within this line representing increased vascularity in granulation tissue.Not observed any distortion of the lining of the femoral head. Based on the radio-morphologic characteristics it is observed, aseptic necrosis of the head of the left femur. Moderate fluid accumulation in the joint. So, the attempt of the repairing process results in a deposition of new bone in the borders of the necrotic area which becomes more and more isolated from the normal living bone. In this stage ( Steinberg stage 2,3 ) a radiolucent lining is seen around the necrotic zone making clear the exact area that has been damaged. The head of the femur still has a normal appearance.

Minor focal distortion of the subchondral surface with aseptic necrosis nature is also seen in the head of the right femur. 
second one two days before the visit. In the first MRI, an altered density in both femoral heads (mainly at left) and osteonecrosis were diagnosed. Between the two MRIs (fig. 2, 3) she was treated with Hyperbaric oxygen therapy (HBO) for 45 times daily (excluding weekends) for $90 \mathrm{~min}$ in 10 meters depth. MRI showed slight deterioration in the findings and the clinical state did not change.

\section{Methods.}

In my private practice we treated the patient with intra-articular injections of MO in the hip joint under ultrasound guidance (Sonosite Micromaxx transducer HFL 50x frequency $15-6 \mathrm{MHz}$ USA,
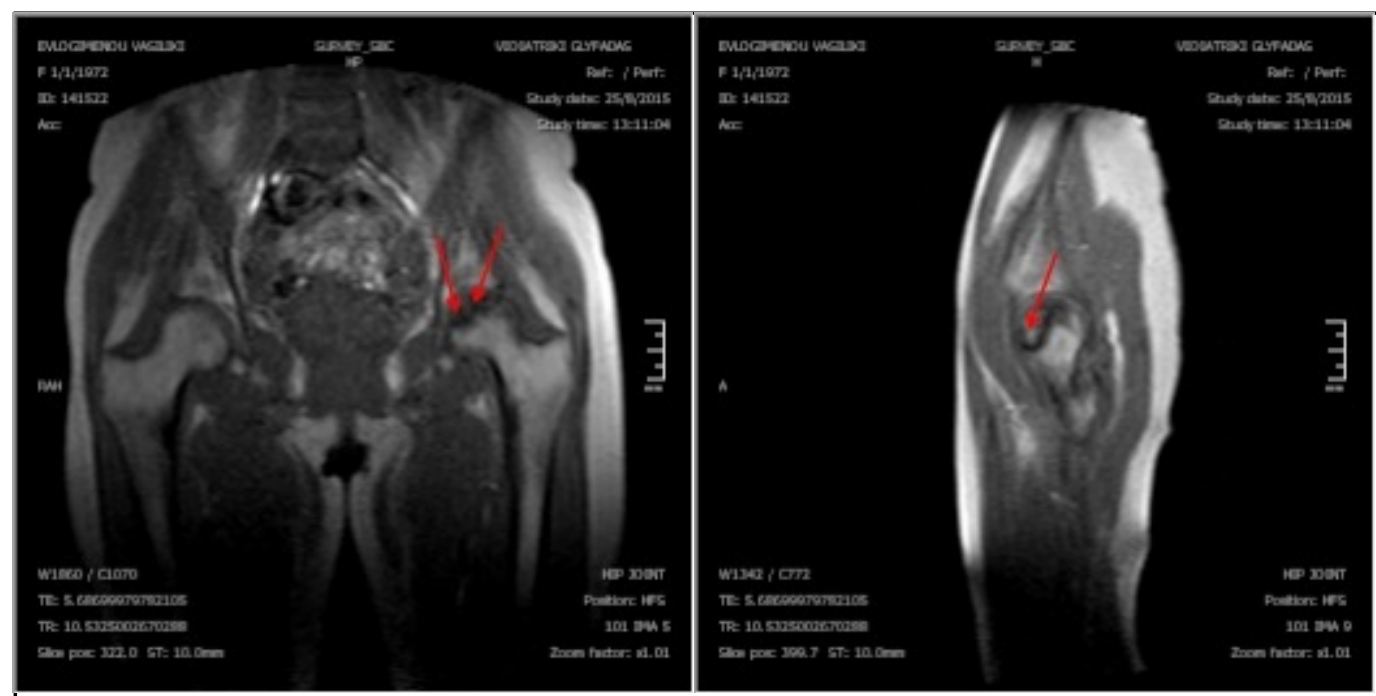

Fig. 2

The noticed sizeable distortion of irregular shape of the subchondral surface of the left femoral head is being rechecked. It is surrounded by a hyper-dense linear margin in T2 image which in the present exam shows moderate amplification after the adminsitration of intravenous contrast agent. As in the previous exam there is no distortion of the lining of the femoral head and the damage is of similar caliber and morphology. There is no deformation of the contour of the femoral head. Based on the radio-morphologic characteristics it is observed, aseptic necrosis of the head of the left femur (arrows).

Minor focal distortion of the subchondral surface with aseptic necrosis nature is also seen in the head of the right femur.
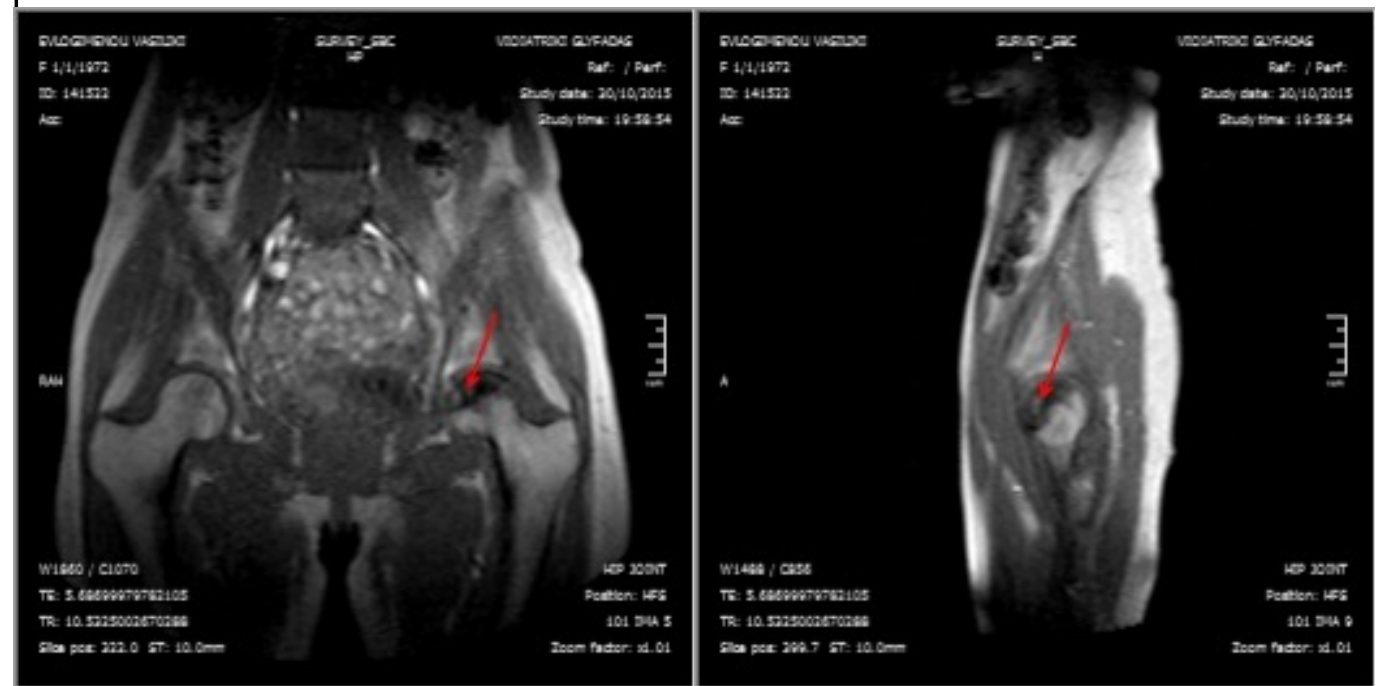

Fig. 3

In comparison to the previous exam, the oedema of limited extent that was present in the head of the left femur is not anymore present while the amount of synovial of fluid is also reduced.

The minor focal distortion of the subchondral surface with aseptic necrosis nature is still present in the head of the right femur (arrows) 
needle Sterican 0.60X60mm D) weekly (fig. 4, 5, 6), in $5 \mathrm{ml}$ volume in $40 \mathrm{mgr}$ concentration $\mathrm{O} 3 / \mathrm{O} 2$ with anterior perpendicular approach. The first two times procaine injection $(1 \mathrm{ml}, 2 \%)$ was used before $\mathrm{O} 3$ injection in order to avoid discomfort from the volume of gas and to relieve the pressure results. Another course of injections with the same concentration and volume was performed in the posterior superior spine for only three times because after this patient was pain free at this point.

\section{RESULTS}

A new MRI of the patient showed that this time, according to the radiologist, the picture of the area was improved with less oedema in the femoral head and less synovial fluid accumulation in the left hip joint. The MRI images of the right hip showed no change between the first second and third. The patient, right after the second injection felt better and enjoyed a night sleep without painkillers. According to our protocol, the course of treatment of the knees consists of seven visits and after the termination of the therapy the pain is relieved and the patient has his movement abilities restored. Additionally, soft weight-bearing of the limb (the patient was using handles) as well as water training were proposed. After 7 visits, the patient was able to walk without support and the last MRI screening showed that the oedema of the suffering area decreased and so did the accumulation of the intra-articular fluid.

\section{DISCUSSION}

Osteonecrosis is a common condition $(15,16,17$, $37)$. It is evident from the previous observations (1, $2,3,4,8)$ that the oxygen ozone therapy is efficient
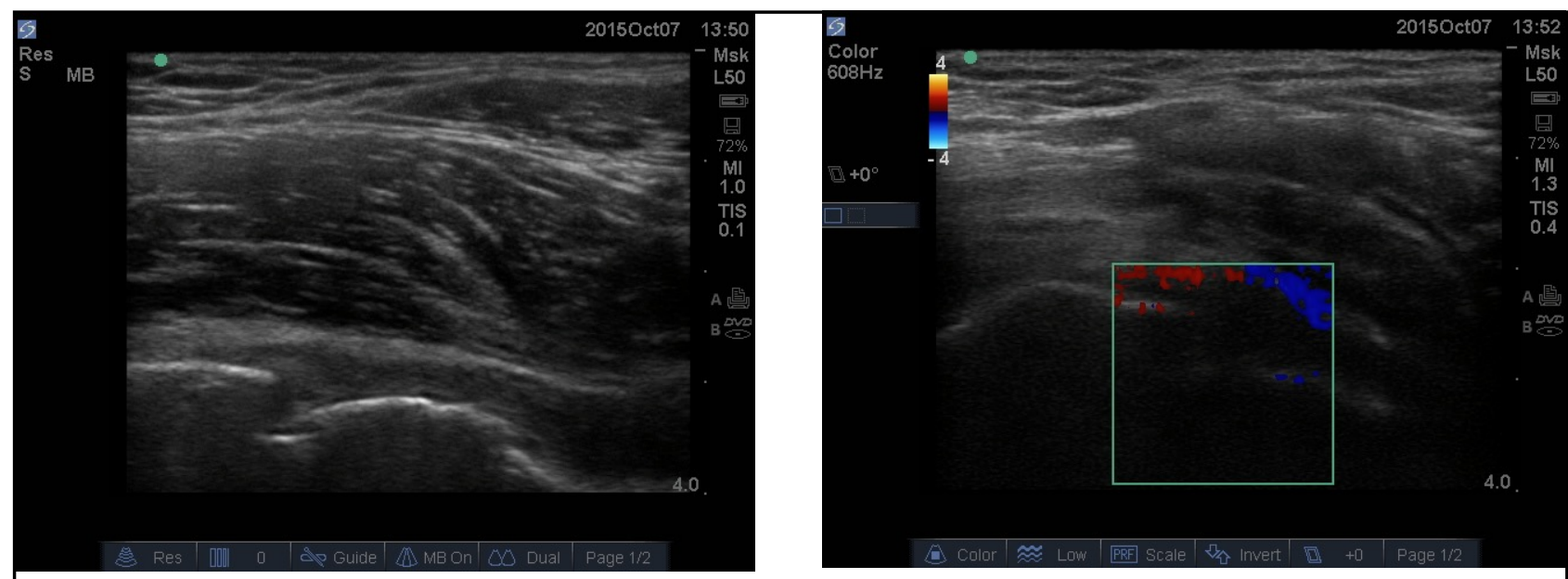

Fig. 4. Before

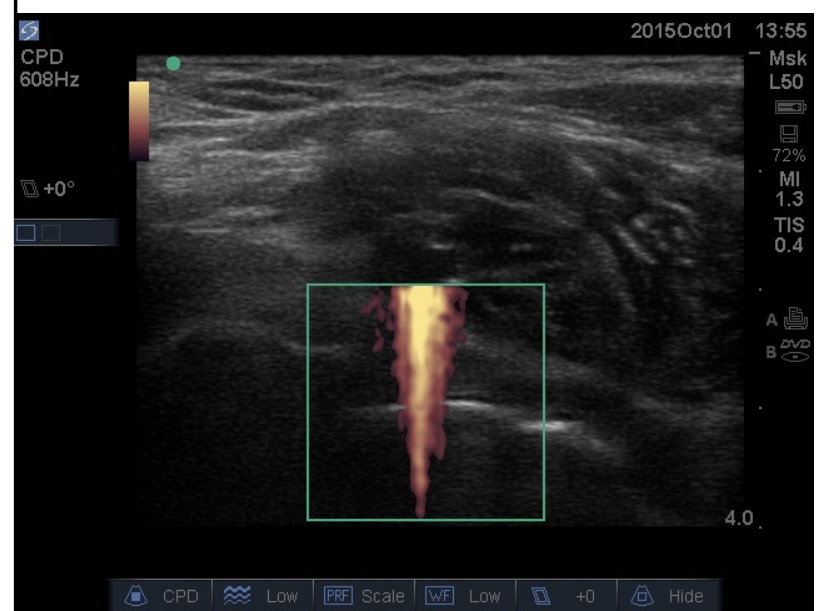

Fig. 5. The injection

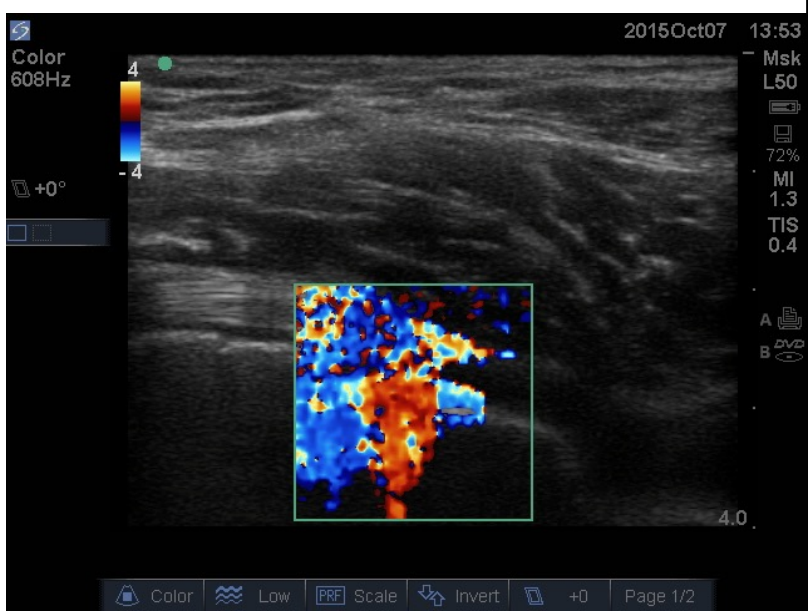

Fig. 6. Four min later 
and gives good results against manifestations associated with oxidative stress like disk herniation and knee arthritis (3) and there are many findings that prove the relationship between oxidative damage of the bone marrow and apoptosis of osteocytes in osteonecrosis of the femoral head (33), recurrent events of oxidative-stress even of minor extent are sufficient to induce osteocyte apoptosis. Acute bone marrow oedema is evident in early MRI (35), consequently, after the intraarticular injection of MO the oedema present on the head of the left femur is reduced (38). Furthermore improve the circulation $(9,10)$ in the femoral head. This evident improvement of the picture of the suffering area proves the efficacy of our therapeutic method.

\section{CONCLUSION}

Judging by the results for the particular condition and study case, seems that the effects of the intraarticular injection of medical ozone were positive even thought the patient didn't respond to hyperbaric oxygen treatment. In the contralateral hip (right), were not observed any notable changes neither after treatment with hyperbaric oxygen nor with ozone in the controlateral hip. Although the study has to be expanded in more cases as well as in cases with different gravity, this therapeutic approach was proved feasible and probably promising and the use of this treatment in humans as well as in animals (39) could be recommended.

\section{REFERENCES}

1. Bonetti M, Fontana A, Cotticelli B, et al. Intraforaminal $\mathrm{O} 2-\mathrm{O} 3$ versus periradicular steroidal infiltrations in lower back pain: randomized controlled study. Am J Neuroradiol. 2005;26:996-1000.

2. Melchionda D, Milillo P, Manente G, Stoppino L, Macarini L. Treatment of radiculopathies: a study of efficacy and tollerability of paravertebral oxygen-ozoneinjections compared with pharmacological anti-inflammatory treatment. J Biol Regul Homeost Agents. 2012;26(3):467-474.

3. Borrelli E, Alexandre A, Iliakis E, Alexandre A, Bocci V. Disc Herniation and Knee Arthritis as Chronic Oxidative Stress Diseases: The Therapeutic Role of Oxygen Ozone Therapy. J Arthritis. 2015;4:161.

4. Chen H, Yu B, Lu C, Lin Q. The effect of intraarticular injection of different concentrations of ozone on the level of TNF- $\alpha$, TNF-R1, and TNFR2 in rats with rheumatoid arthritis. Rheumatol Int. 2013;33(5):1223-1227.

5. Erken HA, Genc O, Erken G, Ayada C, Gundogdu G, Dogan H. Ozone partially prevents diabetic neuropathy in rats. Exp Clin Endocrinol Diabetes. 2015;123(2):101-105.

6. Zhang J, Guan M, Xie C, Luo X, Zhang Q, Xue $Y$. Increased growth factors play a role in wound healing promoted by noninvasive oxygen-ozone therapy in diabetic patients with foot ulcers. Oxid Med Cell Longev. 2014;2014:273475. doi: $10.1155 / 2014 / 273475$.
7. Bocci V, Zanardi I, Huijberts MS, Travagli V. It is time to integrate conventional therapy by ozone therapy in type- 2 diabetes patients. Ann Transl Med. 2014;2(12):117.

8. Leon-Fernandez OS, Pantoja M, Diaz-Soto MT, Dranguet J, Garcia-Insua M, Viebhan-Hansler R, et al. Ozone oxidative post-conditioning reduces oxidative protein damage in patients with disc hernia. Neurol Res. 2012;34(1):59-67.

9. Bocci V. How a calculated oxidative stress can yield multiple therapeutic effects. Free Radic Res. 2012;46(9):1068-1075.

10.Bocci V, Zanardi I, Huijberts MS, Travagli V. Diabetes and chronic oxidative stress. A perspective based on the possible usefulness of ozone therapy. Diabetes Metab Syndr. 2011;5(1): 45-49.

11.Travagli V, Zanardi I, Bocci V. A realistic evaluation of the action of ozone on whole human blood. Int J Biol Macromol. 2006;39(4-5):317-320.

12.Ramesh KS. Management of avascular necrosis of femoral head at pre-collapse stage. Indian $\mathbf{J}$ Orthop. 2009;43(1):6-16.

13.Musso ES, Mitchell SN, Schink-Ascani M, Bassett CA. Results of conservative management of osteonecrosis of the femoral head. A retrospective review. Clin Orthop Relat Res. 1986;207:209-215.

14.Anjan PK, Anusuya D, Quanjun C. Osteonecrosis of the femoral head: An update in year 2012. World J Orthop. 2012;3(5):49-57. 
15.Mankin HJ. Nontraumatic necrosis of bone (osteonecrosis. N Engl J Med. 1992;326:1473-1479.

16.Roshan A, Ram S. The neglected femoral neck fracture in young adults: review of a challenging problem. Clin Med Res. 2008;6:33-39.

17. Min BW, Song KS, Cho CH, Lee SM, Lee KJ. Untreated asymptomatic hips in patients with osteonecrosis of the femoral head. Clin Orthop Relat Res. 2008;466:1087-1092.

18.Calder JD, Buttery L, Revell PA, Pearse M, Polak JM. Apoptosis - a significant cause of bone cell death in osteonecrosis of the femoral head. J Bone Joint Surg Br. 2004;86:1209-1213.

19.Weinstein RS, Nicholas RW, Manolagas SC. Apoptosis of osteocytes in glucocorticoidinduced osteonecrosis of the hip. J Clin Endocrinol Metab. 2000;85:2907-2912.

20.Youm YS, Lee SY, Lee SH. Apoptosis in the osteonecrosis of the femoral head. Clin Orthop Surg. 2010;2:250-255.

21.Bejar J, Peled E, Boss JH. Vasculature deprivation--induced osteonecrosis of the rat femoral head as a model for therapeutic trials. Theor Biol Med Model. 2005;2:24.

22.Assouline-Dayan Y, Chang C, Greenspan A, Shoenfeld Y, Gershwin ME. Pathogenesis and natural history of osteonecrosis. Semin Arthritis Rheum. 2002;32:94-124.

23.Cui Q, Wang GJ, Su CC, Balian G. The Otto Aufranc Award. Lovastatin prevents steroid induced adipogenesis and osteonecrosis. Clin Orthop Relat Res. 1997;344:8-19.

24.Wang GJ, Sweet DE, Reger SI, Thompson RC. Fat-cell changes as a mechanism of avascular necrosis of the femoral head in cortisone-treated rabbits. J Bone Joint Surg Am. 1977;59:729-735.

25.Kannu P, Rielly DD, O'Hyland JC, Kokko LA. Avascular necrosis of the femoral head due to a novel C propeptide mutation in COL2A1. Am J Med Genet A. 2011;155A:1759-1762.

26.Steinberg ME, Steinberg DR. Classification systems for osteonecrosis: an overview. Orthop Clin North Am. 2004;35(3):273-283.

27.Watson RM, Roach NA, Dalinka MK. Avascular necrosis and bone marrow edema syndrome. Radiol Clin North Am. 2004;42(1):207-219.
28.Staudenherz A, Hofmann S, Breitenseher M, Schneider $\mathrm{W}$, Engel AE, Imhof $\mathrm{H}$, et al. Diagnostic patterns for bone marrow oedema syndrome and avascular necrosis of the femoral head in dynamic bone scintigraphy. Nucl Med Commun. 1997;18(12):1178-88.

29.Bocci V. Scientific and medical aspects of ozone therapy. State of the art. Arch Med Res. 2006;37:425-435.

30.Sagai M, Bocci V. Mechanisms of Action Involved in Ozone Therapy: Is healing induced via a mild Oxidative stress. Med Gas Res. 2011;1:29. doi: 10.1186/2045-9912-1-29.

31.Elvis AM, Ekta JS. Ozone therapy: A clinical review. J Nat Sci Biol Med. 2011;2(1):66-70.

32 Zhao ZQ, LiuWL, Gong YL, Bai R, Wang WX . DNA oxidative damage to bone marrow hematopoietic cells and apoptosis of osteocytes in early avascular necrosis of the femoral head induced by glucocorticoids. Chinese Journal of Tissue Engineering Research. 2015;19(11): 1652-1657.

33.Ichiseki, T, Kaneuji A, Kaneko S, Ueda S, Ueda Y, Yonekura H, Fukui K, Matsumoto T . The timing and extent of intraosseous hypoxia in the oxidative stress-induced rat osteonecrosis model. Advances in Bioscience and Biotechnology. 2013;4:814-817.

34.Karantanas AH. Acute bone marrow edema of the hip: role of MR imaging. Eur Radiol. 2007; 17:2225-2228.

35.Ichiseki T, Kaneuji A, Ueda Y, Nakagawa S. Mikami T, Fukui K, Matsumoto T. Osteonecrosis development in a novel rat model characterized by a single application of oxidative stress. Arthritis \& Rheumatism. 2011;63:2138-2141.

36Jones JP. Fat embolism, intravascular coagulation, and osteonecrosis. Clinical Orthopaedics and Related Research. 1993;292(11):294-308.

37.Chan WP, Liu YJ, Huang GS, Jiang CC, Huang $\mathrm{S}$, Chang YC. MRI of joint fluid in femoral head osteonecrosis. Skeletal Radiol. 2002;31(11): 624-630.

38.Piek CJ, Hazewinkel HA, Wolvekamp WT, Nap RC, Mey BP. Long-term follow-up of avascular necrosis of the femoral head in the dog. J Small Anim Pract. 1996;37(1):12-8. 